OPEN ACCESS

Edited by: Julia G. Prado, IrsiCaixa, Spain

Reviewed by: Domenico Mavilio, University of Milan, Italy Paul Goepfert University of Alabama at Birmingham, United States

*Correspondence:

Seik-Soon Khor

seiksoon@gmail.com Katsushi Tokunaga katokunaga@ri.ncgm.go.jp

Specialty section: This article was submitted to Viral Immunology, a section of the journal

Frontiers in Immunology

Received: 26 January 2021 Accepted: 22 March 2021 Published: 22 April 2021

Citation:

Khor SS, Omae Y, Nishida N, Sugiyama M, Kinoshita N, Suzuki T, Suzuki M, Suzuki S, Izumi S, Hojo M,

Ohmagari N, Mizokami M and Tokunaga K (2021) HLAA11:01:01:01, HLA-C12:02:02:01HLA-B52:01:02:02, Age and Sex Are Associated With Severity of Japanese COVID-19 With Respiratory Failure.

Front. Immunol. 12:658570. doi: 10.3389/fimmu.2021.658570

\section{HLA-A*11:01:01:01, HLA- $C *$ 12:02:02:01-HLA-B*52:01:02:02, Age and Sex Are Associated With Severity of Japanese COVID-19 With Respiratory Failure}

\author{
Seik-Soon Khor $^{1 *}$, Yosuke Omae ${ }^{1}$, Nao Nishida ${ }^{2}$, Masaya Sugiyama ${ }^{2}$, Noriko Kinoshita ${ }^{3}$, \\ Tetsuya Suzuki ${ }^{3}$, Michiyo Suzuki ${ }^{3}$, Satoshi Suzuki ${ }^{4}$, Shinyu Izumi ${ }^{5}$, Masayuki Hojo ${ }^{5}$, \\ Norio Ohmagari ${ }^{3}$, Masashi Mizokami ${ }^{2}$ and Katsushi Tokunaga ${ }^{1 *}$

\begin{abstract}
1 Genome Medical Science Project, National Center for Global Health and Medicine Hospital, Tokyo, Japan, ${ }^{2}$ Genome Medical Sciences Project, National Center for Global Health and Medicine, Ichikawa, Japan, ${ }^{3}$ Disease Control and Prevention Center, National Center for Global Health and Medicine Hospital, Tokyo, Japan, ${ }^{4}$ Biobank, National Center for Global Health and Medicine, Tokyo, Japan, ${ }^{5}$ Department of Respiratory Medicine, National Center for Global Health and Medicine Hospital, Tokyo, Japan
\end{abstract}

Severe acute respiratory syndrome coronavirus 2 (SARS-CoV-2), the virus causing coronavirus disease 2019 (COVID-19) was announced as an outbreak by the World Health Organization (WHO) in January 2020 and as a pandemic in March 2020. The majority of infected individuals have experienced no or only mild symptoms, ranging from fully asymptomatic cases to mild pneumonic disease. However, a minority of infected individuals develop severe respiratory symptoms. The objective of this study was to identify susceptible HLA alleles and clinical markers that can be used in risk prediction model for the early identification of severe COVID-19 among hospitalized COVID-19 patients. A total of 137 patients with mild COVID-19 (mCOVID-19) and 53 patients with severe COVID-19 (sCOVID-19) were recruited from the Center Hospital of the National Center for Global Health and Medicine (NCGM), Tokyo, Japan for the period of FebruaryAugust 2020. High-resolution sequencing-based typing for eight HLA genes was performed using next-generation sequencing. In the HLA association studies, HLA$A^{*} 11: 01: 01: 01\left[P_{C}=0.013, O R=2.26(1.27-3.91)\right]$ and HLA-C*12:02:02:01-HLA$B^{*} 52: 01: 01: 02\left[P_{C}=0.020, O R=2.25(1.24-3.92)\right]$ were found to be significantly associated with the severity of COVID-19. After multivariate analysis controlling for other confounding factors and comorbidities, HLA-A*11:01:01:01 [P = 3.34E-03, OR = 3.41 (1.50-7.73)], age at diagnosis $[\mathrm{P}=1.29 \mathrm{E}-02, \mathrm{OR}=1.04(1.01-1.07)]$ and sex at birth $[\mathrm{P}=$ 8.88E-03, OR $=2.92(1.31-6.54)]$ remained significant. The area under the curve of the risk prediction model utilizing HLA-A*11:01:01:01, age at diagnosis, and sex at birth was 0.772 , with sensitivity of 0.715 and specificity of 0.717 . To the best of our knowledge, this is the first article that describes associations of HLA alleles with COVID-19 at the 4-field 
(highest) resolution level. Early identification of potential SCOVID-19 could help clinicians prioritize medical utility and significantly decrease mortality from COVID-19.

Keywords: HLA, allele, haplotype, COVID-19, case-control analysis, susceptibility genes

\section{INTRODUCTION}

Severe acute respiratory syndrome coronavirus 2 (SARS-CoV-2) is the virus responsible for the onset of COVID-19, and has so far infected more than 87 million people worldwide, with the global death toll surpassing 1.8 million (as of January 10, 2021). The majority of infected individuals have experienced only mild or minor symptoms, ranging from asymptomatic cases to mild pneumonic disease. However, a minority of infected individuals developed severe respiratory symptoms but eventually survive with the aid of mechanical ventilatory support.

As of January 10, 2021, mortality rates of COVID-19 (https:// coronavirus.jhu.edu/data/mortality) were reported as highest in Mexico (8.8\%), followed by Iran, Italy, Hungary, and Indonesia with approximate mortality rates of 3-4\%, the United Kingdom, South Africa, Colombia, Canada, Spain, Brazil, France, Poland and Germany at around 2-2.9\%, and other countries with reported mortality rates less than $2 \%$. Differences in mortality rates can be caused by several factors, such as differences in the number of people tested, the capacity of local healthcare systems to handle increasing numbers of patients during the outbreak, the demographics of the population (with mortality rates known to be higher in older populations) and the different preventive measurements attempted by local governments. Nonetheless, while Japan stands as one of the oldest populations in the world (with citizens 65 years or older accounting for approximately $28 \%$ of the total population), the country has only suffered a mortality rate of $1.9 \%$. Emerging clinical evidence has suggested that factors such as Bacillus Calmette-Guérin (BCG)-vaccination status (1), viral genomic strain $(2,3)$ and host genetic factors $(4,5)$ may be related to the severity and mortality of COVID-19.

The immunogenetic background of the host, such as HLA diversity, is well known to play an essential role in determining host responses to emerging infectious diseases such as TB (6), HBV (7-10), HCV (11, 12), HIV (13, 14), SARS-CoV-1 (15-19) and SARS-CoV-2 (20-22). In cases of viral infection, viruses have successfully breached the early layers of the innate defense system (early nonspecific responses) such as fever, phagocytosis and inflammation. The human second line of defense is heavily reliant on the HLA-restricted T-cell response mechanism, in which viral epitopes are presented by dendritic cells to CD8+ T lymphocytes through interactions with HLA class I alleles and CD4+ T lymphocytes through HLA class II alleles. Viral epitope presentation by HLA class I leads to clonal expansion of HLArestricted CD8+ cytotoxic T lymphocytes (CTLs), which are primed to perform antiviral defense during acute infection. Subsequently, latent reinfection and reactivation of the virus are controlled by memory CTLs.
The 2002-2003 SARS-CoV-1 outbreak is known to have higher pathogenicity and higher mortality rates, while SARSCoV-2 infections show higher transmissibility. Experimental HLA association studies have indicated that HLA class I and class II alleles such as $\mathrm{B}^{\star} 46: 01$ (15), $\mathrm{B}^{\star} 07: 03$ (16), $\mathrm{Cw}^{\star} 08: 01$ (17), and DRB1 ${ }^{\star} 12: 02$ (18) carriers are more susceptible to SARS$\mathrm{CoV}-1$ infection. Early studies of SARS-CoV-2 associations with HLA alleles generally used small sample sizes, but $B^{\star} 15: 27$ and $\mathrm{C}^{\star 07: 29}$ have nonetheless been reported as associated with SARS-CoV-2 susceptibility in the Chinese population (20) and a SARS-CoV-2 susceptibility study with larger sample size in China reported associations of $A^{\star} 11: 01, B^{\star} 51: 01$ and $C^{\star} 11: 01$ with severity of COVID-19 in China (23). On the other hand, $\mathrm{B}^{\star} 27: 07, \mathrm{DRB} 1^{\star} 15: 01$, and $\mathrm{DQB} 1^{\star} 06: 02$ have been reported as susceptible HLA alleles in Italian COVID-19 (21). Besides HLA alleles associations, the confounding comorbidities of the host such as older age, smoking, and underlying noncommunicable diseases such as diabetes, hypertension, cardiac disease, chronic lung disease and cancer, have been reported as risk factors for severe COVID-19 according to the most recent (27 May 2020) clinical guidelines from the WHO (https://www.who.int/ publications/i/item/clinical-management-of-covid-19).

Due to emerging evidence that the diversity of HLA allele distributions across different populations is related to the susceptibility to and severity of COVID-19, this study was designed to examine HLA alleles associated with the susceptibility and severity of Japanese COVID-19.

\section{MATERIALS AND METHODS}

\section{National Center for Global Health and Medicine (NCGM) COVID-19 Patients}

A total of 190 Japanese COVID-19 patients (all COVID-19 [aCOVID-19]) were recruited from the Center Hospital of the NCGM for the period of February-August 2020. The biological samples utilized for this research were provided by the Biobank of National Center for Global Health and Medicine (NCGM Biobank) in Japan. In this study, severe COVID-19 (sCOVID19) was defined according to the most recent (27 May 2020) clinical guidelines from the WHO (https://www.who.int/ publications/i/item/clinical-management-of-covid-19), which defined "severe disease" as adults with clinical signs of pneumonia (fever, cough, dyspnea, fast breathing) accompanied by one of the following: respiratory rate $>30$ breaths/min; severe respiratory distress; or peripheral oxygen saturation $\left(\mathrm{SpO}_{2}\right)<90 \%$ on room air. The sCOVID-19 patients comprised $27.9 \%$ of total patients who participated in this study. Written informed consent was obtained from all patients. 


\section{Tokyo Healthy Control (THC)}

THC samples comprised 423 healthy individuals residing in the Tokyo area before the COVID-19 outbreak. Informed consent was obtained from all of the THC sample participants.

\section{High-Resolution HLA Genotyping by Next- Generation Sequencing}

The AllType assay (One Lambda, West Hills, CA) was designed to cover the full length of five HLA genes (HLA-A, -C, -B, -DQA1 and -DPA1) and partial coverage for six HLA genes (HLA-DRB1, -DRB3, -DRB4, -DRB5, -DQB1 and -DPB1). Experimental protocols were carried out following vendor instructions, consisting of HLA target amplification, HLA library preparation, HLA template preparation, and HLA library loading onto an ion 530v1 chip (Thermo Fisher Scientific, Waltham, MA) in the ion chef (Thermo Fisher Scientific) followed by final sequencing on the ion S5 machine (Thermo Fisher Scientific).

\section{HLA Genotype Assignment}

Demultiplexing of barcodes and base-calling was carried out in Torrent Suite version 5.8.0 (Thermo Fisher Scientific). Raw fastq reads were extracted using the FileExporter function in Torrent Suite version 5.8.0. HLA genotype assignments were carried out using two different types of software, namely HLATypeStream Visual (TSV v2.0; One Lambda, West Hills, CA) as the default software for the Alltype ${ }^{\mathrm{TM}}$ NGS Assay and NGSengine ${ }^{\circledR}$ (v2.18.0.17625) by the GenDX company (GenDX, Utrecht, the Netherlands). The default analysis parameters and healthy metrics threshold was applied for TSV2.0 while we applied the "ignore regions" function in the NGSengine to eliminate known sequencing error sites in the ion S5 system. For the fully covered HLA genes (HLA-A, -C, -B, -DQA1 and -DPA1), 4-field HLA alleles were determined after comparing the reads with the IMGT 3.40.0 database; 3-field HLA alleles were determined for partially covered HLA genes (HLA-DRB1, -DRB345, -DQB1 and -DPB1). Novel HLA alleles that are absent from the IMGT 3.40.0 database or ambiguous HLA alleles were subjected to Pacbio Sequel sequencing by H.U. Group Research Institute (Tokyo, Japan). After confirming the presence of novel HLA alleles using Pacbio Sequel sequencing, Pacbio consensus reads were submitted to GenBank and the IMGT nomenclature committee for the official naming of HLA alleles.

\section{Statistical Analysis}

Case-control HLA allele association tests, HLA haplotype estimations, case-control HLA haplotype association tests, Hardy-Weinberg equilibrium tests, and HLA amino acid association tests were prepared and analyzed using the Bridging ImmunoGenomics Data Analysis Workflow Gaps (24) (BIGDAWG) R package. The default parameters of BIGDAWG were used except for the manual specification of HLA haplotypes for testing. Logistic regression, univariate, and multivariate analyses of COVID-19 comorbidities were calculated using R statistics software v4.0.1 (R Foundation for Statistical Computing, Vienna, Austria).
To evaluate associations of risk HLA alleles and comorbidities with the severity of COVID-19, we applied univariate analysis (generalized linear model) to observe the association of associated risk HLA alleles, age, sex, and comorbidities such as high blood pressure, type 2 diabetes, obesity, respiratory diseases (chronic obstructive pulmonary disease [COPD], asthma, tuberculosis $[\mathrm{TB}]$ ), hyperuricemia and dyslipidemia with the severity of COVID-19.

We developed a risk prediction model for identifying potential sCOVID-19 patients. Samples were given a score of 2 for homozygous risk-HLA allele carriers, 1 for heterozygous riskHLA allele carriers, or 0 for non-risk HLA-allele carriers. We created a risk prediction model using unconditional logistic regression in which the respective regression coefficient (weight) is multiplied by the number of risk-HLA alleles carried by the individual and associated factors.

$$
\begin{aligned}
& \log (O R)=\log \left(\frac{p(x)}{1-p(x)}\right) \\
& =\beta_{0}+\beta_{H L A} X_{H L A}+\beta_{\text {Age }} X_{\text {Age }}+\beta_{\text {Sex }} X_{\text {Sex }} \\
& \beta_{0}=\text { intercept }=-4.51671 \\
& \beta_{H L A}=\text { regression coefficient for age }=1.2803 \\
& X_{H L A}=\text { risk HLA allele } \\
& \beta_{\text {Age }}=\text { regression coefficient for age }=0.04763 \\
& X_{\text {Age }}=\text { age at time of infection } \\
& \beta_{\text {Sex }}=\text { regression coefficient for sex }=1.27602 \\
& X_{\text {Sex }}=\text { sex at birth of the individual }
\end{aligned}
$$

The receiver operating characteristic (ROC) curve was plotted using the true-positive rate (sensitivity) against the false-positive rate (1-specificity). The area under the curve (AUC) was used to evaluate the capability of the prediction model to distinguish between sCOVID-19 patients and non-sCOVID-19 patients. Positive predictive value (PPV) and negative predictive value (NPV) were also calculated. These analyses were carried out using RStudio v1.3.1093 (RStudio Team, Boston, MA) with the pROC package v.1.16.2 (25).

\section{RESULTS}

\section{Clinical Characteristics of the 190 Japanese COVID-19 Patients}

Age distribution and other comorbidities in sCOVID-19 and mCOVID-19 patients are shown in Figures 1A, B, respectively. 
A

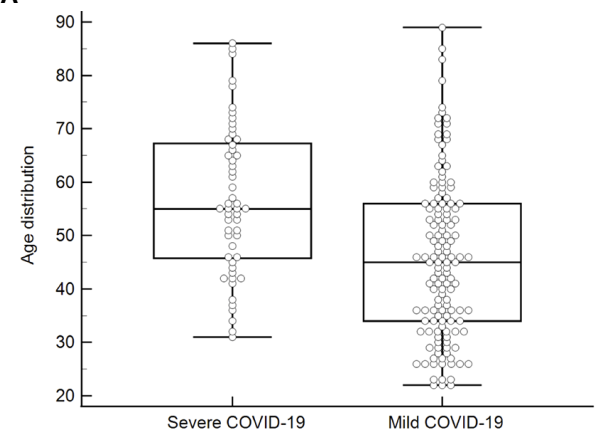

B

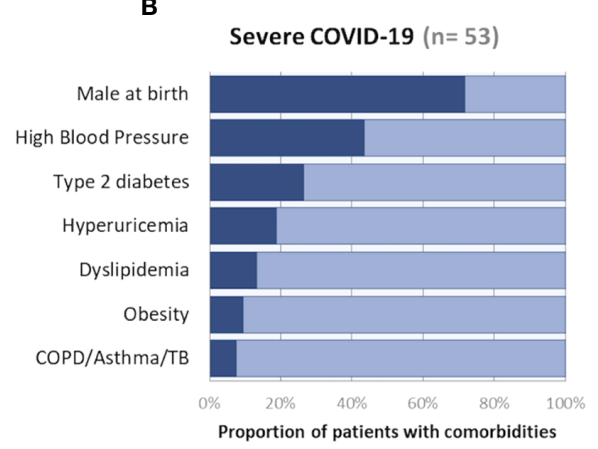

Mild COVID-19 $(n=137)$

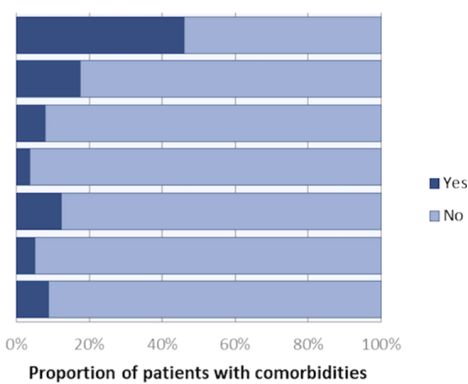

FIGURE 1 | Distributions of age and existing comorbidities in SCOVID-19 patients and mCOVID-19 patients recruited from the Center Hospital of the NCGM for the period of February-August 2020. (A) Box and whiskers graph showing the age distribution of NCGM sCOVID-19 patients and mCOVID-19 patients with a mean age at diagnosis for sCOVID-19 patients and mCOVID-19 patients of 57 years and 46 years, respectively. (B) Proportion of patients with comorbidities (male at birth, high blood pressure, type 2 diabetes, hyperuricemia, dyslipidemia, obesity and COPD/asthma/TB) in NCGM sCOVID-19 patients and mCOVID-19 patients.

Mean age at diagnosis for SCOVID-19 and mCOVID-19 were 57 years and 46 years, respectively, with males comprising $72 \%$ of sCOVID-19 patients and $46 \%$ of mCOVID-19 patients. In general, the most common comorbidities in sCOVID-19 were high blood pressure $(43 \%, 23 / 53)$, type 2 diabetes $(26 \%, 14 / 53)$, hyperuricemia $(19 \%, 10 / 53)$, dyslipidemia $(13 \%, 7 / 53)$, obesity $(9 \%, 5 / 53)$ and COPD/asthma/TB $(8 \%, 4 / 53)$. In comparison, comorbidities were shown to be lower in MCOVID-19 with high blood pressure $(18 \%, 24 / 137)$, type 2 diabetes $(8 \%, 11 / 137)$, hyperuricemia $(4 \%, 5 / 137)$, dyslipidemia $(12 \%, 17 / 137)$, obesity (5\%, 7/137) and COPD/Asthma/TB (9\%, 12/137).

A comprehensive comparison of HLA allele associations for four different subgroups was performed in this study, namely: 1) aCOVID-19 versus healthy controls; 2) mCOVID-19 vs. healthy controls; 3) sCOVID-19 vs. healthy controls; and 4) sCOVID-19 vs. mCOVID-19.

\section{No Association of HLA Alleles With All COVID-19 (aCOVID-19 vs. Controls)}

For the identification of HLA alleles associated with SARS-CoV-2 infection, all 190 aCOVID-19 patients (comprising both mCOVID19 and sCOVID-19) were compared with the 423 Japanese healthy controls. None of the HLA alleles in the 8 HLA genes tested showed significance after multiple corrections (Supplementary Table 1).

\section{No Association of HLA Alleles With Mild COVID-19 (mCOVID-19 vs. Controls)}

To identify potential HLA alleles associated with mild symptoms of COVID-19, the 137 mCOVID-19 patients were compared with the healthy controls. No HLA alleles remained significant after multiple corrections (Supplementary Table 2). This result indicated that HLA frequencies for mCOVID-19 were similar to those of healthy controls.

\section{Associations of HLA Alleles With Severe COVID-19 (sCOVID-19 vs. Controls)}

Severity of COVID-19 is directly related to the management of COVID-19 infection and also contributes to mortality from COVID-19. Association studies of the 8 HLA genes were carried out by comparing HLA allele frequencies in the 53 sCOVID-19 patients and 423 Japanese healthy controls (Table 1): HLA-A ${ }^{\star} 11: 01: 01: 01\left[\mathrm{P}_{\mathrm{c}}=0.013, \mathrm{OR}=2.26(1.27-3.91)\right]$, HLA-C $^{\star} 12: 02: 02: 01\left[\mathrm{P}_{\mathrm{c}}=0.043, \mathrm{OR}=2.13(1.18-3.71)\right]$ and HLA-B $^{\star} 52: 01: 01: 02\left[\mathrm{P}_{\mathrm{c}}=0.021, \mathrm{OR}=2.22(1.22-3.87)\right]$ were significantly associated with the severity of COVID-19 after multiple corrections. To determine the independence of associated HLA alleles, HLA haplotype analyses were carried out. A total of 4 haplotypes remained significant after multiple corrections (Table 2), namely HLA-A*24:02:01:01HLA-B ${ }^{\star} 52: 01: 01: 02\left[\mathrm{P}_{\mathrm{c}}=0.039, \mathrm{OR}=2.06(1.11-3.67)\right]$, HLA$\mathrm{A}^{\star} 24: 02: 01-\mathrm{HLA}-\mathrm{C}^{\star} 12: 02: 02: 01\left[\mathrm{P}_{\mathrm{c}}=0.039, \mathrm{OR}=2.06(1.11-\right.$ 3.67)], HLA- $C^{\star} 12: 02: 02: 01-H L A-B^{\star} 52: 01: 01: 02\left[\mathrm{P}_{\mathrm{c}}=0.020\right.$, $\mathrm{OR}=2.25(1.24-3.92)]$ and HLA-A ${ }^{\star} 24: 02: 01: 01-\mathrm{HLA}-$ $\mathrm{C}^{\star}$ 12:02:01:01-HLA-B 52:01:01:02 $\left[\mathrm{P}_{\mathrm{c}}=0.033, \mathrm{OR}=2.09\right.$ (1.12-3.72)]. None of the HLA class II haplotypes or HLA class I - HLA class II haplotypes remained significant after multiple corrections (Supplementary Table 3). The most significantly associated HLA haplotype was HLA$\mathrm{C}^{\star}$ 12:02:02:01-HLA-B ${ }^{\star}$ 2:01:01:02 $\left[\mathrm{P}_{\mathrm{c}}=0.020, \mathrm{OR}=2.25\right.$ (1.24-3.92)], and the level of significance decreased after including protective HLA- ${ }^{\star} 24: 02: 01: 01\left[\mathrm{P}_{\mathrm{c}}=0.251, \mathrm{OR}=\right.$ $0.78(0.49-1.22)]$ into the abovementioned haplotype, HLA$\mathrm{A}^{\star}$ 24:02:01:01-HLA-C ${ }^{\star}$ 12:02:01:01-HLA-B ${ }^{\star}$ 52:01:01:02 $\left[\mathrm{P}_{\mathrm{c}}=\right.$ $0.033, \mathrm{OR}=2.09(1.12-3.72)]$. Subsequent conditional analysis (Table 3) was performed on the statistically significant HLA alleles, HLA-A*11:01:01:01, HLA-C ${ }^{\star} 12: 02: 02: 01$ and HLA$B^{\star} 52: 01: 01: 02$. Each of HLA-A ${ }^{\star} 11: 01: 01: 01$ and HLA$C^{\star} 12: 02: 02: 01$ and HLA-B ${ }^{\star 52: 01: 01: 02}$ were found to be independently associated with severity of COVID-19. All HLA- 
TABLE 1 | Association analysis of HLA-A, -C, -B, -DRB1, -DQA1, -DQB1, -DPA1 and -DPB1 alleles in 53 Japanese severe CoVID-19 patients and 423 Japanese healthy controls

\begin{tabular}{|c|c|c|c|c|c|c|c|c|c|c|c|c|c|c|c|}
\hline HLA & $\begin{array}{l}\text { 2-field } \\
\text { Allele }\end{array}$ & Control\% & sCovid\% & OR $(95 \% \mathrm{Cl})$ & Pc-value & $\begin{array}{l}\text { 3-field } \\
\text { Allele }\end{array}$ & Control\% & sCovid\% & OR $(95 \% \mathrm{Cl})$ & Pc-value & $\begin{array}{l}\text { 4-field } \\
\text { Allele }\end{array}$ & Control\% & scovID\% & OR (95\% Cl) & Pc-value \\
\hline A & 02:01 & 10.2 & 9.4 & $0.92(0.41-1.85)$ & NS & 02:01:01 & 10.2 & 9.4 & $0.92(0.41-1.85)$ & NS & 02:01:01:01 & 10.2 & 9.4 & $0.92(0.41-1.85)$ & NS \\
\hline A & 02:06 & 7.1 & 6.6 & $0.93(0.35-2.11)$ & NS & 02:06:01 & 7.1 & 6.6 & $0.93(0.35-2.11)$ & NS & 02:06:01:01 & 5.9 & 6.6 & $1.13(0.42-2.59)$ & NS \\
\hline A & $11: 01$ & 9.8 & 19.8 & $2.26(1.27-3.91)$ & 0.015 & 11:01:01 & 9.8 & 19.8 & $2.26(1.27-3.91)$ & 0.013 & 11:01:01:01 & 9.8 & 19.8 & $2.26(1.27-3.91)$ & 0.013 \\
\hline A & $24: 02$ & 37.2 & 31.1 & $0.76(0.48-1.20)$ & NS & 24:02:01 & 37.2 & 31.1 & $0.76(0.48-1.20)$ & NS & 24:02:01:01 & 36.8 & 31.1 & $0.78(0.49-1.22)$ & NS \\
\hline A & $26: 01$ & 7.8 & 4.7 & $0.59(0.18-1.49)$ & NS & & & & & & & & & & \\
\hline A & $31: 01$ & 7.8 & 11.3 & $1.52(0.72-2.96)$ & NS & 31:01:02 & 7.8 & 11.3 & $1.52(0.72-2.96)$ & NS & $31: 01: 02: 01$ & 7.8 & 11.3 & $1.52(0.72-2.96)$ & NS \\
\hline A & 33:03 & 9.3 & 10.4 & $1.13(0.53-2.24)$ & NS & 33:03:01 & 9.3 & 10.4 & $1.13(0.53-2.24)$ & NS & 33:03:01:01 & 9.3 & 10.4 & $1.13(0.53-2.24)$ & NS \\
\hline A & binned & 11.0 & 6.6 & $0.57(0.22-1.28)$ & NS & binned & 11.0 & 6.6 & $0.57(0.22-1.28)$ & NS & binned & 12.5 & 6.6 & $0.49(0.19-1.10)$ & NS \\
\hline C & 01:02 & 17.4 & 10.4 & $0.55(0.26-1.06)$ & NS & 01:02:01 & 17.4 & 10.4 & $0.55(0.26-1.06)$ & NS & 01:02:01:05 & 8.0 & 6.6 & $0.81(0.31-1.84)$ & NS \\
\hline C & 03:03 & 13.2 & 10.4 & $0.76(0.36-1.48)$ & NS & 03:03:01 & 13.2 & 10.4 & $0.76(0.36-1.48)$ & NS & 03:03:01:01 & 12.7 & 10.4 & $0.79(0.37-1.55)$ & NS \\
\hline C & 03:04 & 12.2 & 12.3 & $1.01(0.50-1.90)$ & NS & 03:04:01 & 12.2 & 12.3 & $1.01(0.50-1.90)$ & NS & 03:04:01:02 & 12.2 & 12.3 & $1.01(0.50-1.90)$ & NS \\
\hline C & 07:02 & 14.2 & 13.2 & $0.92(0.47-1.68)$ & NS & 07:02:01 & 14.2 & 13.2 & $0.92(0.47-1.68)$ & NS & 07:02:01:01 & 5.6 & 7.5 & $1.39(0.55-3.07)$ & NS \\
\hline C & 08:01 & 5.7 & 8.5 & $1.54(0.65-3.30)$ & NS & 08:01:01 & 5.7 & 8.5 & $1.54(0.65-3.30)$ & NS & 08:01:01:01 & 5.7 & 8.5 & 1.54 (0.65-3.30) & NS \\
\hline C & $12: 02$ & 9.8 & 18.9 & $2.13(1.18-3.71)$ & 0.043 & 12:02:02 & 9.8 & 18.9 & $2.13(1.18-3.71)$ & 0.043 & $12: 02: 02: 01$ & 9.8 & 18.9 & $2.13(1.18-3.71)$ & 0.043 \\
\hline C & 14:02 & 5.8 & 6.6 & $1.15(0.43-2.65)$ & NS & 14:02:01 & 5.8 & 6.6 & $1.15(0.43-2.65)$ & NS & 14:02:01:01 & 5.8 & 6.6 & $1.15(0.43-2.65)$ & NS \\
\hline C & $14: 03$ & 8.3 & 6.6 & $0.78(0.29-1.75)$ & NS & 14:03:01 & 8.3 & 6.6 & $0.78(0.29-1.75)$ & NS & 14:03:01:01 & 8.3 & 6.6 & $0.78(0.29-1.75)$ & NS \\
\hline C & binned & 13.4 & 13.2 & $0.98(0.50-1.80)$ & NS & binned & 13.4 & 13.2 & $0.98(0.50-1.80)$ & NS & binned & 16.4 & 16.0 & $0.97(0.53-1.71)$ & NS \\
\hline B & $15: 01$ & 8.9 & 10.4 & $1.18(0.55-2.34)$ & NS & 15:01:01 & 8.9 & 10.4 & $1.18(0.55-2.34)$ & NS & 15:01:01:01 & 8.9 & 10.4 & $1.18(0.55-2.34)$ & NS \\
\hline B & $35: 01$ & 7.6 & 8.5 & $1.12(0.48-2.36)$ & NS & $35: 01: 01$ & 7.6 & 8.5 & $1.12(0.48-2.36)$ & NS & $35: 01: 01: 02$ & 7.1 & 7.5 & $1.07(0.43-2.34)$ & NS \\
\hline B & $40: 01$ & 5.4 & 8.5 & $1.61(0.67-3.46)$ & NS & 40:01:02 & 5.4 & 8.5 & $1.61(0.67-3.46)$ & NS & & & & & \\
\hline B & 44:03 & 8.2 & 7.5 & $0.91(0.37-1.97)$ & NS & 44:03:01 & 8.2 & 6.6 & $0.79(0.30-1.78)$ & NS & 44:03:01:10 & 8.2 & 6.6 & $0.79(0.30-1.78)$ & NS \\
\hline B & $51: 01$ & 8.3 & 8.5 & $1.02(0.43-2.13)$ & NS & $51: 01: 01$ & 8.3 & 8.5 & $1.02(0.43-2.13)$ & NS & 51:01:01:01 & 6.0 & 5.7 & $0.94(0.32-2.26)$ & NS \\
\hline B & $52: 01$ & 9.6 & 18.9 & $2.19(1.21-3.81)$ & 0.028 & $52: 01: 01$ & 9.6 & 18.9 & $2.19(1.21-3.81)$ & 0.028 & $52: 01: 01: 02$ & 9.5 & 18.9 & $2.22(1.22-3.87)$ & 0.021 \\
\hline B & $54: 01$ & 8.2 & 6.6 & $0.79(0.30-1.78)$ & NS & 54:01:01 & 8.2 & 6.6 & 0.79 (0.30-1.78) & NS & $54: 01: 01: 01$ & 8.2 & 6.6 & $0.79(0.30-1.78)$ & NS \\
\hline B & binned & 30.3 & 23.6 & $0.71(0.42-1.15)$ & NS & binned & 30.3 & 24.5 & $0.75(0.45-1.21)$ & NS & binned & 34.4 & 28.3 & $0.75(0.47-1.19)$ & NS \\
\hline DRB1 & 04:05 & 14.5 & 11.3 & $0.75(0.37-1.43)$ & NS & 04:05:01 & 14.5 & 11.3 & $0.75(0.37-1.43)$ & NS & 04:05:01:01 & 14.2 & 11.3 & $0.77(0.37-1.46)$ & NS \\
\hline DRB1 & 08:03 & 7.6 & 4.7 & $0.60(0.18-1.52)$ & NS & & & & & & & & & & \\
\hline DRB1 & 09:01 & 15.5 & 11.3 & $0.70(0.34-1.32)$ & NS & 09:01:02 & 15.5 & 11.3 & $0.70(0.34-1.32)$ & NS & 09:01:02:01 & 15.5 & 11.3 & $0.70(0.34-1.32)$ & NS \\
\hline DRB1 & $12: 01$ & 5.3 & 6.6 & $1.26(0.47-2.91)$ & NS & 12:01:01 & 5.3 & 6.6 & $1.26(0.47-2.91)$ & NS & & & & & \\
\hline DRB1 & $13: 02$ & 8.0 & 8.5 & $1.07(0.45-2.24)$ & NS & 13:02:01 & 8.0 & 8.5 & $1.07(0.45-2.24)$ & NS & 13:02:01:02 & 7.6 & 7.5 & $0.99(0.40-2.14)$ & NS \\
\hline DRB1 & 15:01 & 8.0 & 5.7 & $0.69(0.24-1.64)$ & NS & 15:01:01 & 8.0 & 5.7 & $0.69(0.24-1.64)$ & NS & & & & & \\
\hline DRB1 & 15:02 & 8.3 & 16.0 & $2.10(1.11-3.80)$ & NS & 15:02:01 & 8.3 & 16.0 & $2.10(1.11-3.80)$ & NS & & & & & \\
\hline DRB1 & binned & 25.9 & 33.0 & $1.41(0.89-2.21)$ & NS & binned & 25.9 & 33.0 & $1.41(0.89-2.21)$ & NS & binned & 33.4 & 51.9 & $2.15(1.40-3.29)$ & 0.001 \\
\hline DQA1 & 01:02 & 16.0 & 17.0 & $1.08(0.59-1.87)$ & NS & 01:02:01 & 15.7 & 14.2 & $0.88(0.46-1.59)$ & NS & 01:02:01:01 & 7.6 & 5.7 & $0.73(0.25-1.73)$ & NS \\
\hline DQA1 & & & & & & & & & & & 01:02:01:08 & 7.4 & 7.5 & $1.02(0.41-2.22)$ & NS \\
\hline DQA1 & 01:03 & 16.6 & 20.8 & $1.32(0.76-2.22)$ & NS & 01:03:01 & 16.6 & 20.8 & $1.32(0.76-2.22)$ & NS & 01:03:01:01 & 8.3 & 16.0 & $2.10(1.11-3.80)$ & NS \\
\hline DQA1 & 01:04 & 5.2 & 8.5 & $1.69(0.70-3.64)$ & NS & 01:04:01 & 5.2 & 8.5 & $1.69(0.70-3.64)$ & NS & 01:04:01:01 & 5.2 & 8.5 & 1.69 (0.70-3.64) & NS \\
\hline DQA1 & 03:01 & 8.1 & 7.5 & $0.93(0.37-2.01)$ & NS & 03:01:01 & 8.1 & 7.5 & $0.93(0.37-2.01)$ & NS & 03:01:01:01 & 8.1 & 7.5 & $0.93(0.37-2.01)$ & NS \\
\hline DQA1 & 03:02 & 16.7 & 14.2 & $0.82(0.43-1.48)$ & NS & 03:02:01 & 16.7 & 14.2 & $0.82(0.43-1.48)$ & NS & 03:02:01:01 & 12.4 & 12.3 & $0.99(0.49-1.85)$ & NS \\
\hline DQA1 & 03:03 & 17.0 & 14.2 & $0.80(0.42-1.45)$ & NS & 03:03:01 & 17.0 & 14.2 & $0.80(0.42-1.45)$ & NS & 03:03:01:03 & 14.5 & 11.3 & $0.75(0.37-1.43)$ & NS \\
\hline DQA1 & binned & 13.8 & 15.1 & $1.11(0.59-1.99)$ & NS & binned & 14.0 & 17.9 & $1.34(0.74-2.32)$ & NS & binned & 24.0 & 27.4 & $1.20(0.73-1.91)$ & NS \\
\hline DQB1 & 03:01 & 11.8 & 8.5 & $0.69(0.30-1.43)$ & NS & 03:01:01 & 11.8 & 8.5 & $0.69(0.30-1.43)$ & NS & & & & & \\
\hline
\end{tabular}




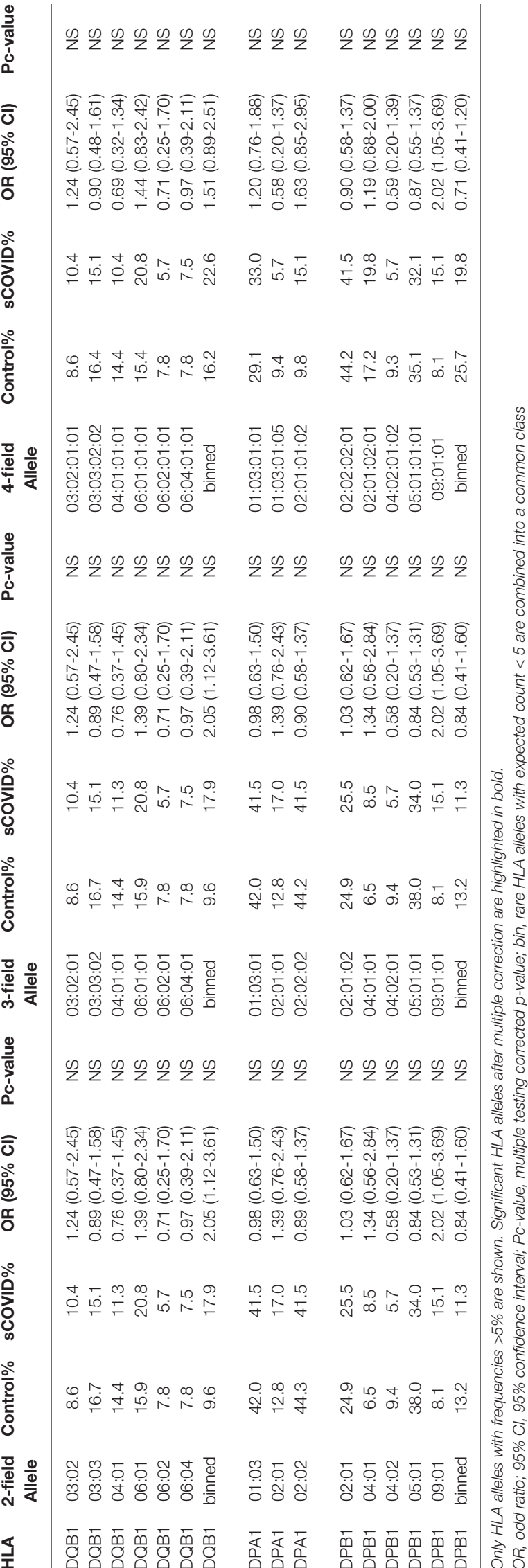

$\mathrm{C}^{\star}$ 12:02:02:01 alleles were shown to be tightly linked with HLA$\mathrm{B}^{\star}$ 52:01:01:02 alleles and only a small proportion of $A^{\star}$ 11:01:01:01 alleles were linked with HLA-C*12:02:02:01 and HLA-B ${ }^{\star 52: 01: 01: 02 ~ h a p l o t y p e s ~(F i g u r e ~ 2) . ~}$

\section{Associations of HLA Alleles With Severe COVID-19 (sCOVID-19 vs. mCOVID-19)}

To identify potential HLA markers that can be used to prioritize treatment for early hospitalized COVID-19 patients, we compared HLA allele frequencies between the 53 sCOVID-19 patients and the 137 mCOVID-19 patients (Supplementary Table 4). HLA-A ${ }^{\star} 11: 01: 01: 01\left[\mathrm{P}_{\mathrm{c}}=0.022\right.$, OR $=2.57(1.29-$ 5.09)] carrier was significantly associated with increased severity of COVID-19 patients, while due to the smaller effect size, HLA$\mathrm{C}^{\star} 12: 02: 02: 01[\mathrm{P}=0.067, \mathrm{OR}=1.76(0.90-3.36)]$ and HLA$\mathrm{B}^{\star}$ 52:01:01:02 $[\mathrm{P}=0.067, \mathrm{OR}=1.76(0.90-3.36)]$ showed lower significance in comparison to the 53 sCOVID-19 patients and 423 Japanese healthy controls (Table 1).

\section{Risk Prediction Model Using Associated HLA alleles and Comorbidities}

To evaluate the association of HLA-A ${ }^{\star} 11: 01: 01: 01$ and HLA$C^{\star}$ 12:02:02:01- HLA-B ${ }^{\star}$ 52:01:01:02 haplotypes with various comorbidities, we performed univariate logistic regression analyses (Table 4) and observed that HLA-A*11:01:01:01 $[\mathrm{P}=$ $1.73 \mathrm{E}-03, \mathrm{OR}=3.09(1.52-6.25)]$, age $[\mathrm{P}=3.52 \mathrm{E}-05, \mathrm{OR}=1.05$ (1.03-1.07)], sex $[\mathrm{P}=1.83 \mathrm{E}-03, \mathrm{OR}=2.96(1.50-5.91)]$, high blood pressure $[\mathrm{P}=3.22 \mathrm{E}-04, \mathrm{OR}=3.61(1.79-7.26)]$, type 2 diabetes $[\mathrm{P}=1.40 \mathrm{E}-03, \mathrm{OR}=4.11(1.73-9.79)]$, and hyperuricemia $[\mathrm{P}=1.60 \mathrm{E}-03, \mathrm{OR}=6.14(1.99-18.96)]$ are significantly associated with the severity of COVID-19 after Bonferroni correction (threshold $=0.05 / 10$ comorbidities $=$ 0.05). After multivariate analysis controlling for other confounding factors and comorbidities, HLA-A ${ }^{*} 11: 01: 01: 01$ $[\mathrm{P}=3.34 \mathrm{E}-03, \mathrm{OR}=3.41(1.50-7.73)]$, age $[\mathrm{P}=1.29 \mathrm{E}-02$, $\mathrm{OR}=1.04(1.01-1.07)]$ and $\operatorname{sex}[\mathrm{P}=8.88 \mathrm{E}-03, \mathrm{OR}=2.92$ (1.31-6.54)] remained significant.

The AUC of a risk prediction model that includes HLA$A^{\star} 11: 01: 01: 01$, age, and sex was 0.772 , with sensitivity of 0.715 and specificity of 0.717 (Figure 3). PPV and NPV are illustrated in Figure 4, evaluating the distribution of cases and controls against $\log (\mathrm{OR})$ values. The threshold of the plot was calculated from the optimal sensitivity and specificity in the AUC. A significant difference ( $\mathrm{P}=5.22 \mathrm{E}-08)$ was observed between risk and non-risk of case-control in this study, with an odds ratio of 6.37 (95\%CI $=3.15-12.86)$.

\section{DISCUSSION}

We conducted a high-resolution sequencing-based typing of eight HLA genes to evaluate the association of HLA alleles/ haplotypes with SARS-CoV-2 infection susceptibility and the 
TABLE 2 | Comprehensive HLA haplotype analysis of HLA-A, -C and -B in Japanese severe COVID-19 and Japanese healthy control.

\begin{tabular}{|c|c|c|c|c|c|c|}
\hline Locus & Allele & Control $(2 n=846) \%$ & Severe_COVID $(2 n=106) \%$ & OR $(95 \% \mathrm{Cl})$ & p.value & p.corrected \\
\hline$A \sim C \sim B$ & 24:02:01:01 07:02:01:03 07:02:01:01 & 5.8 & 0.9 & $0.16(0.00-0.93)$ & 0.035 & NS \\
\hline$A \sim C \sim B$ & 24:02:01:01 12:02:02:01 52:01:01:02 & 8.9 & 17.0 & $2.09(1.12-3.72)$ & 0.008 & 0.033 \\
\hline$A \sim C \sim B$ & 33:03:01:01 14:03:01:01 44:03:01:10 & 7.6 & 6.6 & $0.85(0.32-1.93)$ & 0.703 & NS \\
\hline$A \sim C \sim B$ & binned & 77.7 & 75.5 & $0.89(0.54-1.48)$ & 0.611 & NS \\
\hline $\mathrm{C} \sim \mathrm{B}$ & 01:02:01:05 54:01:01:01 & 7.9 & 6.6 & $0.83(0.31-1.87)$ & 0.645 & NS \\
\hline $\mathrm{C} \sim \mathrm{B}$ & 03:03:01:01 35:01:01:02 & 5.9 & 4.7 & $0.79(0.24-2.03)$ & 0.621 & NS \\
\hline $\mathrm{C} \sim \mathrm{B}$ & 03:04:01:02 40:02:01:01 & 5.2 & 3.8 & $0.71(0.18-2.02)$ & 0.524 & NS \\
\hline$C \sim B$ & 07:02:01:03 07:02:01:01 & 6.8 & 3.8 & $0.54(0.14-1.49)$ & 0.228 & NS \\
\hline C $\sim B$ & 12:02:02:01 52:01:01:02 & 9.4 & 18.9 & 2.25 (1.24-3.92) & 0.003 & 0.020 \\
\hline$C \sim B$ & 14:02:01:01 51:01:01:01 & 5.8 & 5.7 & $0.98(0.33-2.36)$ & 0.958 & NS \\
\hline$C \sim B$ & 14:03:01:01 44:03:01:10 & 8.2 & 6.6 & $0.79(0.30-1.78)$ & 0.564 & NS \\
\hline$C \sim B$ & binned & 50.8 & 50.0 & $0.97(0.63-1.48)$ & 0.875 & NS \\
\hline$A \sim C$ & 24:02:01:01 07:02:01:03 & 5.8 & 0.9 & $0.16(0.00-0.93)$ & 0.035 & NS \\
\hline$A \sim C$ & 24:02:01:01 12:02:02:01 & 9.0 & 17.0 & 2.06 (1.11-3.67) & 0.010 & 0.039 \\
\hline$A \sim C$ & 33:03:01:01 14:03:01:01 & 7.8 & 6.6 & $0.84(0.32-1.90)$ & 0.674 & NS \\
\hline$A \sim C$ & binned & 77.4 & 75.5 & $0.90(0.55-1.50)$ & 0.650 & NS \\
\hline$A \sim B$ & 24:02:01:01 07:02:01:01 & 5.9 & 0.9 & $0.15(0.00-0.91)$ & 0.032 & NS \\
\hline$A \sim B$ & 24:02:01:01 52:01:01:02 & 9.0 & 17.0 & $2.06(1.11-3.67)$ & 0.010 & 0.039 \\
\hline$A \sim B$ & 33:03:01:01 44:03:01:10 & 7.6 & 6.6 & $0.85(0.32-1.93)$ & 0.703 & NS \\
\hline$A \sim B$ & binned & 77.4 & 75.5 & $0.90(0.55-1.50)$ & 0.650 & NS \\
\hline
\end{tabular}

Significant HLA haplotypes after multiple correction are highlighted in bold.

OR, odd ratio; $95 \% \mathrm{Cl}$, 95\% confidence interval; PC-value, multiple testing corrected p-value; binned, rare HLA alleles with expected count < 5 are combined into a common class

TABLE 3 | Conditional analysis on top associated HLA alleles and HLA haplotypes with the severity of COVID-19.

\begin{tabular}{llll}
\hline HLA alleles & \multicolumn{1}{c}{ Condition on } & SE & P-value \\
\hline$A^{*}$ 11:01:01:01 & $C^{\star} 12: 02: 02: 01$ & 0.825 & 0.002 \\
& $B^{\star} 52: 01: 01: 02$ & 0.830 & 0.002 \\
$B^{\star} 52: 01: 01: 02$ & $A^{*} 11: 01: 01: 01$ & 0.874 & 0.004 \\
& $C^{*} 12: 02: 02: 01$ & 0.133 & 0.840 \\
$C^{*} 12: 02: 02: 01$ & $A^{\star} 11: 01: 01: 01$ & 0.879 & 0.003 \\
& $B^{*} 52: 01: 01: 02$ & 0.686 & 0.279 \\
$A^{*} 11: 01: 01: 01$ & $C^{*} 12: 02: 02: 01-B^{*} 52: 01: 01: 02$ & 0.833 & 0.002 \\
\hline
\end{tabular}

SE, standard error.

severity of COVID-19. A total of 53 sCOVID-19 patients and 137 mCOVID-19 patients were recruited from the NCGM, Tokyo, Japan, from February to August 2020 and 423 previously recruited healthy controls were used as healthy comparators in this study.

To evaluate HLA alleles potentially associated with susceptibility to SARS-CoV-2 infection, aCOVID-19 (Supplementary Table 1) and mCOVID-19 (Supplementary Table 2) were compared with healthy controls, respectively. We found that no HLA alleles remained significant after Bonferroni correction and there was no disease-specific in the HLA genes, suggesting that no HLA alleles were either protective or conferred susceptibility to SARS-CoV-2 infection.

Patients with sCOVID-19 are tightly associated with COVID19 mortality. We observed that the HLA-A*11:01:01:01 $\left[\mathrm{P}_{\mathrm{c}}=\right.$ 0.013, OR $=2.26(1.27-3.91)]$ allele and HLA-C ${ }^{\star} 12: 02: 02: 01-$ $\mathrm{HLA}^{\star}{ }^{\star}$ 52:01:01:02 $\left[\mathrm{P}_{\mathrm{c}}=0.020, \mathrm{OR}=2.25(1.24-3.92)\right]$ haplotype were significantly associated with the severity of COVID-19. A recent publication by Wang et al. (23) reported significant associations of HLA-A ${ }^{\star} 11: 01, H L A-B^{\star} 51: 01$, and
HLA-C ${ }^{\star} 14: 02$ with severe or critically severe Chinese COVID19 patients compared to mild or moderate Chinese COVID-19 patients. Accordingly, HLA-A ${ }^{\star} 11: 01$ was significantly associated with both Japanese and Chinese sCOVID-19 patients, while HLA-C ${ }^{\star}$ 12:02:02:01-HLA-B ${ }^{\star 52: 01: 01: 02 ~ a n d ~ H L A-B ~}{ }^{\star 51: 01-~}$ HLA- $C^{\star} 14: 02$ were associated in Japanese and Chinese, respectively. The HLA associations of HLA-A ${ }^{\star} 11: 01$ and HLA$C^{\star}$ 12:02-HLA-B ${ }^{\star 52: 01}$ are of particular importance, as Asian populations carry relatively high frequencies of these HLA alleles. Referring to the Allele Frequency Net Database (AFND) 2020 (26), HLA-C ${ }^{\star} 12: 02-H_{L A}-B^{\star} 52: 01$ is commonly present in Japanese with a frequency of $10.5 \%$, followed by Asian populations residing in the United States (1.5-3.4\%). On the other hand, HLA-A ${ }^{\star} 11: 01$ is predominant in South-east Asia (17.7-61.3\%), Japan (8.2-11.1\%), China (16.2-61.3\%) and the Oceania region $(2.6-63.6 \%)$. We expect that HLA-A ${ }^{\star} 11: 01$ and HLA-C ${ }^{\star} 12: 02-H L A-B^{\star} 52: 01$ could potentially act as a predictive marker for the severity of COVID-19 in the Asia region.

However, we could not replicate results for $B^{\star} 46: 01$, which has been predicted to have the fewest predicted binding peptides for SARS-CoV-2, thus Individuals carrying $B^{\star} 46: 01$ are predicted to be more vulnerable to SARS-CoV-2 infection (27). Further, we could not confirm the findings of an HLA-binding prediction study (3) that predicted HLA-A ${ }^{\star} 11: 01, \mathrm{HLA}_{-}{ }^{\star} 02: 06$, and HLA$B^{\star}$ 54:01 as protective against SARS-CoV-2. The two studies mentioned above are based solely on the imperfect HLA prediction algorithm between host HLA alleles and SARSCoV-2 sequences; besides, it should be noted that peptides predicted with high binding scores do not necessarily imply immunogenicity from the host.

Subsequently, we have developed a risk prediction model (Figures 3, 4) that includes HLA-A ${ }^{\star} 11: 01: 01: 01$ carrier status, age, and sex for the early detection of potential sCOVID-19 

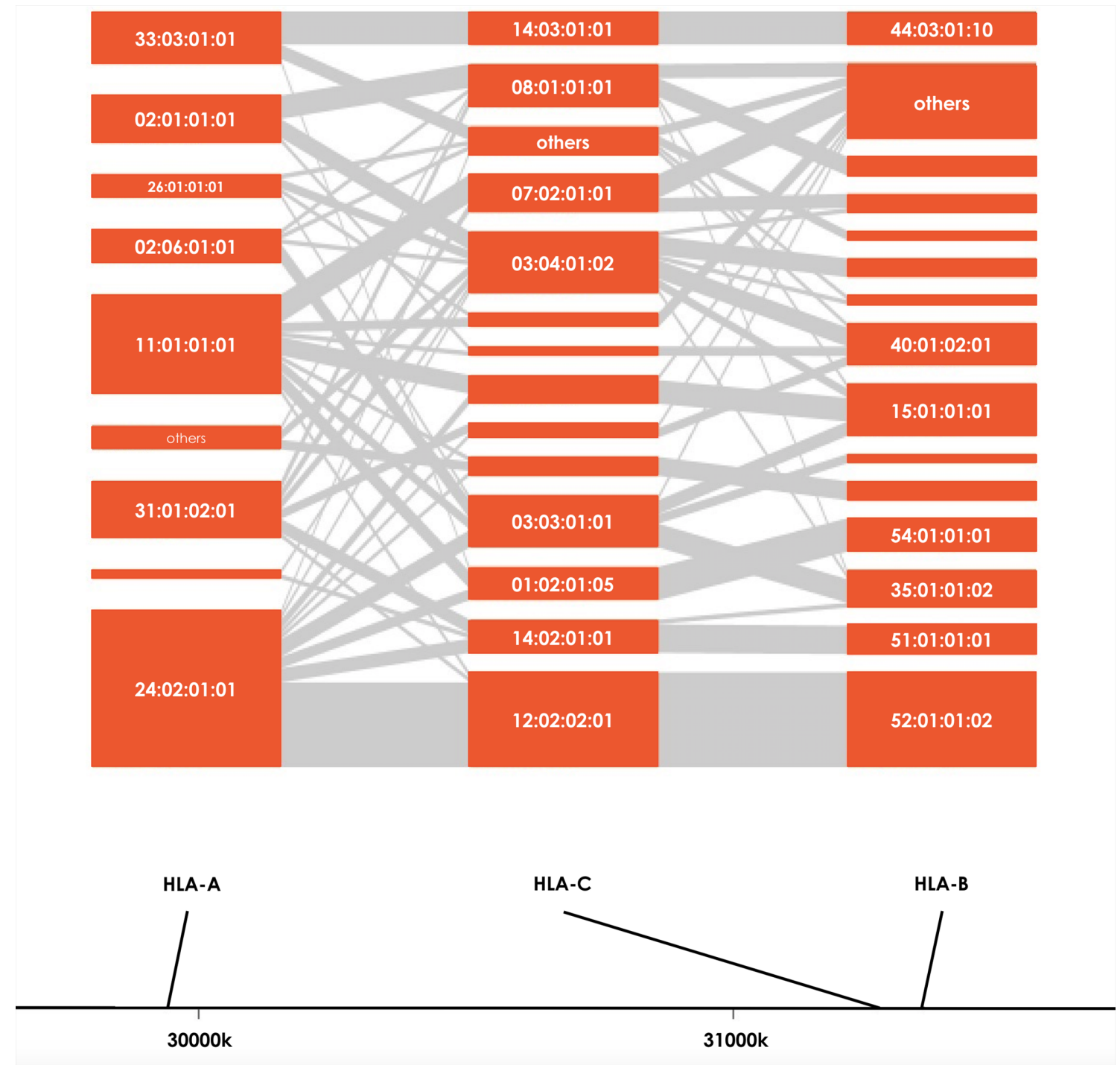

FIGURE 2 | HLA haplotype distributions of HLA-A, HLA-B and HLA-C in sCOVID-19 patients. The height of each orange boxes represents the number of individuals carrying the specific HLA alleles and the grey interconnecting lines showing the proportion of individual haplotypes.

TABLE 4 | Univariate analysis (Generalized Linear Model) of associated HLA alleles and comorbidities with the severity of COVID-19.

\begin{tabular}{|c|c|c|c|c|}
\hline Factors & Univariate $p$-value & OR (95\% Cl) & Multivariate $\mathrm{p}$-value & OR (95\% Cl) \\
\hline$A^{*} 11: 01: 01: 01$ & 1.71E-03 & 3.09 (1.52-6.25) & 3.34E-03 & $3.41(1.50-7.73)$ \\
\hline$C^{\star} 12: 02: 02: 01-B^{\star} 52: 01: 01: 02$ & 7.42E-02 & $1.75(0.95-3.22)$ & - & - \\
\hline Age & 3.52E-05 & $1.05(1.03-1.07)$ & $1.29 \mathrm{E}-02$ & $1.04(1.01-1.07)$ \\
\hline Sex & $1.83 E-03$ & $2.96(1.50-5.91)$ & 8.88E-03 & $2.92(1.31-6.54)$ \\
\hline High_Blood_Pressure & 3.22E-04 & $3.61(1.79-7.26)$ & $5.25 E-01$ & $1.33(0.55-3.25)$ \\
\hline Type2Diabetes & $1.40 \mathrm{E}-03$ & $4.11(1.73-9.79)$ & $1.00 \mathrm{E}-01$ & $2.30(0.85-6.18)$ \\
\hline Obesity & 2.79E-01 & $1.93(0.56-6.39)$ & - & - \\
\hline COPD_Asthma_TB & $7.88 \mathrm{E}-01$ & $0.85(0.26-2.76)$ & - & - \\
\hline Hyperuricemia & $1.60 \mathrm{E}-03$ & $6.14(1.99-18.96)$ & 7.93E-02 & $3.04(0.88-10.50)$ \\
\hline Dyslipidemia & 8.82E-01 & $1.07(0.42-2.76)$ & - & - \\
\hline
\end{tabular}

Bold text represents the statistically significant comorbidities in each testing models. 


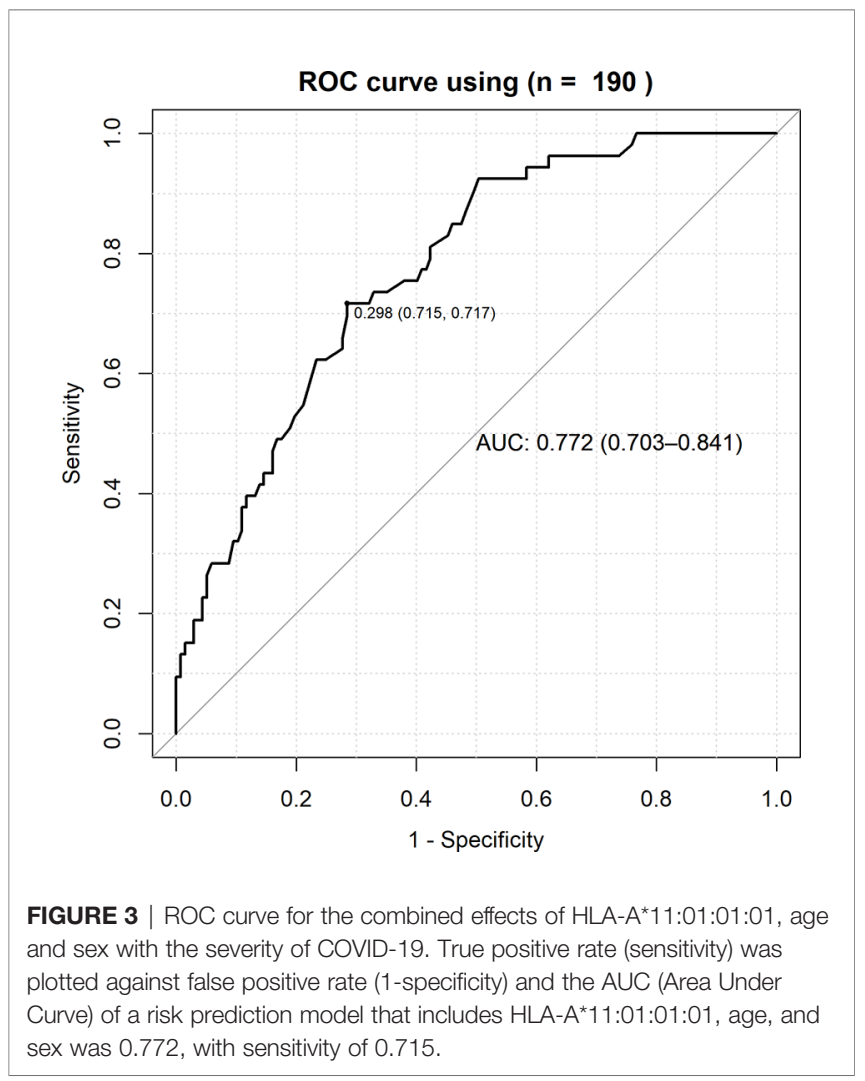

patients in the hospital. The AUC value of the risk prediction model was 0.772 with optimal sensitivity of 0.715 and specificity of 0.717 , suggesting that the current prediction model could be further improved by incorporating additional clinical and genetic factors associated with the severity of COVID-19. However, this study demonstrated that the contributions of genetic factors and comorbidities are useful in identifying potential sCOVID-19 cases. Further investigation and validation are needed to confirm the applicability of the prediction model to a bigger sample in Japan and the generalizability of this predictive model to other Asian populations.

All samples used in this study were collected in the Center Hospital of the NCGM for the period from February to August 2020, where the mutant strain of SARS-CoV-2 was not yet prevalent in Japan. However, further studies are needed to evaluate correlations between the emerging mutant strain of SARS-CoV-2 and severity of COVID-19.

\section{DATA AVAILABILITY STATEMENT}

The datasets presented in this study can be found in HLA COVID-19 repositories (http://www.hlacovid19.org/).

\section{ETHICS STATEMENT}

The studies involving human participants were reviewed and approved by National Center for Global Health and Medicine.

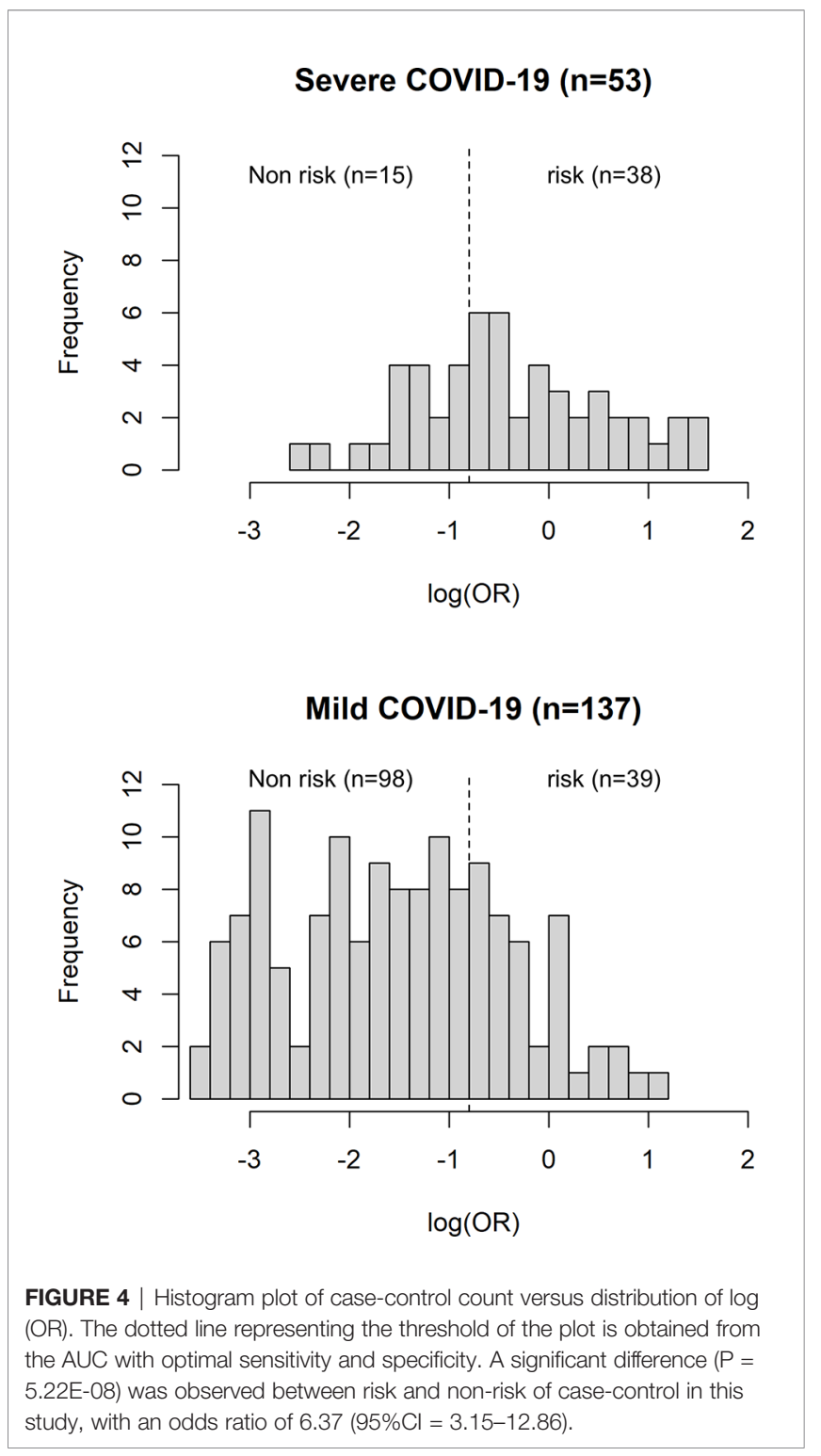

The patients/participants provided their written informed consent to participate in this study.

\section{AUTHOR CONTRIBUTIONS}

S-SK and KT contributed to the writing of the manuscript. YO, $\mathrm{NN}, \mathrm{MaS}$, and S-SK contributed to the experimental aspect of this study. NK, TS, MiS, SS, SI, MH, NO, and MM contributed to the sample collection of this study. All authors contributed to the article and approved the submitted version.

\section{FUNDING}

This research is supported by Japan Agency for Medical Research and Development (AMED) under Grant Number 
JP20kk0205012 and JP20fk0108104 and the NCGM Intramural Research Fund 20A2002D.

\section{ACKNOWLEDGMENTS}

We express our gratitude to Ms. Yoshimi Shigemori and Ms. Ayumi Nakayama for their technical assistance. We thank Dr. Ikue Ito and Dr. Tetsuya Sato from H.U. Group Research Institute for their technical support for Pacbio Sequel

\section{REFERENCES}

1. Berg MK, Yu Q, Salvador CE, Melani I, Kitayama S. Mandated Bacillus Calmette-Guérin (BCG) vaccination predicts flattened curves for the spread of COVID-19. Sci Adv (2020) 6(32):eabc1463. doi: 10.1126/sciadv.abc1463

2. Islam MR, Hoque MN, Rahman MS, Alam ASMR, Akther M, Puspo JA, et al. Genome-wide analysis of SARS-CoV-2 virus strains circulating worldwide implicates heterogeneity. Sci Rep (2020) 10(1):14004. doi: 10.1038/s41598020-70812-6

3. Toyoshima Y, Nemoto K, Matsumoto S, Nakamura Y, Kiyotani K. SARSCoV-2 genomic variations associated with mortality rate of COVID-19. J Hum Genet (2020). doi: 10.1038/s10038-020-0808-9

4. Initiative C-HG. The COVID-19 Host Genetics Initiative, a global initiative to elucidate the role of host genetic factors in susceptibility and severity of the SARSCoV-2 virus pandemic. Eur J Hum Genet (2020) 28(6):715-8. doi: 10.1038/ s41431-020-0636-6

5. Ellinghaus D, Degenhardt F, Bujanda L, Buti M, Albillos A, Invernizzi P, et al. Genomewide Association Study of Severe Covid-19 with Respiratory Failure. N Engl J Med (2020). doi: 10.1056/NEJMoa2020283

6. Toyo-Oka L, Mahasirimongkol S, Yanai H, Mushiroda T, Wattanapokayakit $\mathrm{S}$, Wichukchinda N, et al. Strain-based HLA association analysis identified HLA-DRB1*09:01 associated with modern strain tuberculosis. HLA (2017) 90 (3):149-56. doi: 10.1111/tan.13070

7. Nishida N, Ohashi J, Khor SS, Sugiyama M, Tsuchiura T, Sawai H, et al. Understanding of HLA-conferred susceptibility to chronic hepatitis B infection requires HLA genotyping-based association analysis. Sci Rep (2016) 6:24767. doi: 10.1038/srep24767

8. Sawai H, Nishida N, Khor SS, Honda M, Sugiyama M, Baba N, et al. Genomewide association study identified new susceptible genetic variants in HLA class I region for hepatitis B virus-related hepatocellular carcinoma. Sci Rep (2018) 8(1):7958. doi: 10.1038/s41598-018-26217-7

9. Nishida N, Sugiyama M, Sawai H, Nishina S, Sakai A, Ohashi J, et al. Key HLA-DRB1-DQB1 haplotypes and role of the BTNL2 gene for response to a hepatitis B vaccine. Hepatology (2018) 68(3):848-58. doi: 10.1002/hep.29876

10. Huang YH, Liao SF, Khor SS, Lin YJ, Chen HY, Chang YH, et al. Large-scale genome-wide association study identifies HLA class II variants associated with chronic HBV infection: a study from Taiwan Biobank. Aliment Pharmacol Ther (2020) 52(4):682-91. doi: 10.1111/apt.15887

11. Lee MH, Huang YH, Chen HY, Khor SS, Chang YH, Lin YJ, et al. Human leukocyte antigen variants and risk of hepatocellular carcinoma modified by hepatitis $\mathrm{C}$ virus genotypes: A genome-wide association study. Hepatology (2018) 67(2):651-61. doi: 10.1002/hep.29531

12. Catamo E, Zupin L, Freato N, Polesello V, Celsi F, Crocé SL, et al. HLA-G regulatory polymorphisms are associated with susceptibility to HCV infection. HLA (2017) 89(3):135-42. doi: 10.1111/tan.12959

13. Pereyra F, Jia X, McLaren PJ, Telenti A, de Bakker PI, Walker BD, et al. The major genetic determinants of HIV-1 control affect HLA class I peptide presentation. Science (2010) 330(6010):1551-7. doi: 10.1126/science.1195271

14. Park YJ, Etemad B, Ahmed H, Naranbhai V, Aga E, Bosch RJ, et al. Impact of HLA Class I Alleles on Timing of HIV Rebound After Antiretroviral Treatment Interruption. Pathog Immun (2017) 2(3):431-45. doi: 10.20411/pai.v2i3.222

15. Lin M, Tseng HK, Trejaut JA, Lee HL, Loo JH, Chu CC, et al. Association of HLA class I with severe acute respiratory syndrome coronavirus infection. BMC Med Genet (2003) 4:9. doi: 10.1186/1471-2350-4-9 sequencing. We would like to thank NCGM Biobank for providing the study materials, clinical information, and technical supports.

\section{SUPPLEMENTARY MATERIAL}

The Supplementary Material for this article can be found online at: https://www.frontiersin.org/articles/10.3389/fimmu.2021. 658570/full\#supplementary-material

16. Ng MH, Lau KM, Li L, Cheng SH, Chan WY, Hui PK, et al. Association of humanleukocyte-antigen class I $\left(\mathrm{B}^{\star} 0703\right)$ and class II $\left(\mathrm{DRB1}{ }^{\star} 0301\right)$ genotypes with susceptibility and resistance to the development of severe acute respiratory syndrome. J Infect Dis (2004) 190(3):515-8. doi: 10.1086/421523

17. Chen YM, Liang SY, Shih YP, Chen CY, Lee YM, Chang L, et al. Epidemiological and genetic correlates of severe acute respiratory syndrome coronavirus infection in the hospital with the highest nosocomial infection rate in Taiwan in 2003. J Clin Microbiol (2006) 44(2):359-65. doi: 10.1128/JCM.44.2.359-365.2006

18. Keicho N, Itoyama $\mathrm{S}$, Kashiwase $\mathrm{K}$, Phi NC, Long HT, Ha LD, et al. Association of human leukocyte antigen class II alleles with severe acute respiratory syndrome in the Vietnamese population. Hum Immunol (2009) 70 (7):527-31. doi: 10.1016/j.humimm.2009.05.006

19. Wang SF, Chen KH, Chen M, Li WY, Chen YJ, Tsao CH, et al. Humanleukocyte antigen class I Cw 1502 and class II DR 0301 genotypes are associated with resistance to severe acute respiratory syndrome (SARS) infection. Viral Immunol (2011) 24(5):421-6. doi: 10.1089/vim.2011.0024

20. Wang W, Zhang W, Zhang J, He J, Zhu F. Distribution of HLA allele frequencies in 82 Chinese individuals with coronavirus disease-2019 (COVID-19). HLA (2020b) 96(2):194-6. doi: 10.1111/tan.13941

21. Novelli A, Andreani M, Biancolella M, Liberatoscioli L, Passarelli C, Colona VL, et al. HLA allele frequencies and susceptibility to COVID-19 in a group of 99 Italian patients. HLA (2020) 96(5):610-4. doi: 10.1111/tan.14047

22. Amoroso A, Magistroni P, Vespasiano F, Bella A, Bellino S, Puoti F, et al. HLA and AB0 Polymorphisms May Influence SARS-CoV-2 Infection and COVID19 Severity. Transplantation (2020). doi: 10.1097/TP.0000000000003507

23. Wang F, Huang S, Gao R, Zhou Y, Lai C, Li Z, et al. Initial whole-genome sequencing and analysis of the host genetic contribution to COVID-19 severity and susceptibility. Cell Discov (2020a) 6(1):83. doi: 10.1038/s41421-020-00231-4

24. Pappas DJ, Marin W, Hollenbach JA, Mack SJ. Bridging ImmunoGenomic Data Analysis Workflow Gaps (BIGDAWG): An integrated case-control analysis pipeline. Hum Immunol (2016) 77(3):283-7. doi: 10.1016/ j.humimm.2015.12.006

25. Robin X, Turck N, Hainard A, Tiberti N, Lisacek F, Sanchez, JC, et al. pROC: an open-source package for $\mathrm{R}$ and $\mathrm{S}+$ to analyze and compare ROC curves. BMC Bioinf (2011) 12:77. doi: 10.1186/1471-2105-12-77

26. Gonzalez-Galarza FF, McCabe A, Santos EJMD, Jones J, Takeshita L, OrtegaRivera ND, et al. Allele frequency net database (AFND) 2020 update: goldstandard data classification, open access genotype data and new query tools. Nucleic Acids Res (2020) 48(D1):D783-8. doi: 10.1093/nar/gkz1029

27. Nguyen A, David JK, Maden SK, Wood MA, Weeder BR, Nellore A, et al. Human Leukocyte Antigen Susceptibility Map for Severe Acute Respiratory Syndrome Coronavirus 2. J Virol (2020) 94(13). doi: 10.1128/JVI.00510-20

Conflict of Interest: The authors declare that the research was conducted in the absence of any commercial or financial relationships that could be construed as a potential conflict of interest.

Copyright (C) 2021 Khor, Omae, Nishida, Sugiyama, Kinoshita, Suzuki, Suzuki, Suzuki, Izumi, Hojo, Ohmagari, Mizokami and Tokunaga. This is an open-access article distributed under the terms of the Creative Commons Attribution License (CC BY). The use, distribution or reproduction in other forums is permitted, provided the original author(s) and the copyright owner(s) are credited and that the original publication in this journal is cited, in accordance with accepted academic practice. No use, distribution or reproduction is permitted which does not comply with these terms. 